Die Schweizerische Gesellschaft für Allgemeine Innere Medizin (SGIM) beschäftigte sich in den letzten zwei Jahren u.a. mit der Frage, welcher Nutzen für Patienten durch einzelne Untersuchungen und Behandlungen entsteht. Erstmals publiziert die SGIM nun eine Liste von internistischen Tests und Behandlungen, die Patienten in der Regel wenig Nutzen bringen und daher allenfalls verzichtbar sind. Eine solche Überprüfung ärztlicher Tätigkeiten durch medizinische Fachgesellschaften ermöglicht Verbesserungen im Sinne des Patientenwohls. Die FMH unterstützt diese Initiative.

Dr. med. Jürg Schlup, Präsident der FMH

\title{
Die SGIM startet ihre Kampagne «Smarter Medicine» mit der ersten «Top-5-Liste» der Schweiz
}

Während der Platin-Symposien der Schweizerischen Gesellschaft für Allgemeine Innere Medizin (SGIM) in Thun in den Jahren 2012 und 2013 hat eine Reihe von Referenten, Vertretern von ambulant und privat praktizierenden Ärzten, Spitälern, Universitäten, FMH, BAG, pharmazeutischer Industrie, Krankenkassen, Politik und Medien über das Thema «Kann weniger mehr sein?» diskutiert.

Dieses Thema ist zentraler Gegenstand der «ChoosingWisely-Initiative» [1], die 2012 in den USA ins Leben gerufen wurde, und von NICE (National Institute of Health and Clinical Excellence, United Kingdom) das 1999 [2] in Grossbritannien startete. Beide Projekte wurden nach kurzer Zeit in Australien [3], Italien und anderen Ländern aufgegriffen. In diesem Rahmen versuchten zahlreiche medizinische Disziplinen, eine sogenannte «Top-5-Liste» zu erstellen. Dabei handelt es sich um fünf wissenschaftlich erwiesene Untersuchungen und Therapien, die es zu vermeiden gilt. Obwohl sie den Patienten keinen messbaren (oder nur einen geringen) Nutzen bieten und ein potenzielles Risiko von Nebenwirkungen bergen (vor allem bei Zusatzuntersuchungen oder Eingriffen, zu denen sie irrtümlich führen können), werden sie oft von den betroffenen Fachärztinnen und Fachärzten verschrieben.

Nach einem regen Meinungsaustausch zu diesem Thema in Thun sah sich die SGIM bereit, diese Herausforderung anzunehmen und als Pionierin ein Pendant der «Choosing WiselyInitiative» für die Schweiz zu lancieren. Die mit «Smarter Medicine» betitelte Initiative orientiert sich am Positionspapier der Schweizerischen Akademie der Medizinischen Wissenschaften zum Thema «Ein nachhaltiges Gesundheitssystem

\section{Die medizinischen Verfahren in der \\ Schweiz sind zwar «smart»; aber geht es noch etwas «smarter»?}

für die Schweiz» [4]. Zudem nimmt sie weitere, weltweit angestellte Überlegungen zur medizinischen Überversorgung unter dem Titel «Too much medicine» [5] auf.

Ein Artikel dieser Ausgabe präsentiert die erste Top-5-Liste der SGIM, die den Bereich der ambulanten Allgemeinen Inneren Medizin betrifft. Die nächste Top-5-Liste wird im kommen- den Jahr veröffentlicht und beschäftigt sich mit Eingriffen, die im Rahmen der stationären Allgemeinen Inneren Medizin durchgeführt werden.

Wie Sie diesen Artikeln entnehmen können, zeigt sich die SGIM respektvoll gegenüber den Ärzten und den Patienten. Davon zeugt der Name der Kampagne, «Smarter Medicine»: Die medizinischen Verfahren in der Schweiz sind zwar «smart»; aber geht es noch etwas «smarter»? Dazu hat die SGIM Empfehlungen zu Strategien ausgesprochen, die vermieden werden sollten, sofern der klinische Zustand oder die individuelle Situation des Patienten sowie seine Beziehung zu seinem Arzt

\section{«To choose wisely» setzt einen Dialog} zwischen Arzt und Patient voraus.

nichts Anderes erfordern. Letztlich zielt es darauf ab, Entscheidungen zu fördern, die von Ärzten und Patienten gemeinsam getroffen werden.

«To choose wisely» setzt genau das voraus: den Dialog zwischen Arzt und Patient. Für die Ärzte bedeutet dies manchmal das Erlernen geeigneter Gesprächstechniken, für die Patienten die Übernahme einer gewissen Verantwortung für ihre Erkrankung. Auf Ebene der Gesundheitssysteme bedarf es hier eines breit abgestützten Konsenses und einer aufwändigen Medienkommunikation. Der Anspruch der SGIM ist es, einen positiven Beitrag zu diesen Bemühungen und Debatten zu leisten.

Prof. Dr. med. Jean-Michel Gaspoz, Vorsitzender des Präsidiums der Schweizerischen Gesellschaft für Allgemeine Innere Medizin

1 Choosing wisely: an initiative of the ABIM Foundation 2013. (www.choosingwisely.org).

2 Sarah Garner. Disinvestment form low value clinical interventions: NICEly done? BMJ. 2011;343:d4519 doi: 10.1136.

3 Elshaug AG, et al. Over 150 potentially low-value health care practices: an Australian study. MJA. 2012;197:556-60.

4 Un système de santé durable pour la Suisse. Feuille de route des Académies suisses des sciences. Académies suisses des sciences; 2012.

5 Glasziou P, et al. Too much medicine; too little care. Time to wind back the harms of overdiagnosis and overtreatment. BMJ. 2013;347:f4247. doi: 10.1136. 